\title{
Anharmonic Nuclear Motion and the Relative Stability of Hexagonal and Cubic ice
}

\author{
Edgar A. Engel, Bartomeu Monserrat, and Richard J. Needs \\ TCM Group, Cavendish Laboratory, University of Cambridge, \\ J. J. Thomson Avenue, Cambridge CB3 OHE, United Kingdom \\ (Received 30 January 2015; published 24 June 2015)
}

\begin{abstract}
We use extensive first-principles quantum mechanical calculations to show that, although the static lattice and harmonic vibrational energies are almost identical, the anharmonic vibrational energy of hexagonal ice is significantly lower than that of cubic ice. This difference in anharmonicity is crucial, stabilizing hexagonal ice compared with cubic ice by at least $1.4 \mathrm{meV} / \mathrm{H}_{2} \mathrm{O}$, in agreement with experimental estimates. The difference in anharmonicity arises predominantly from molecular $\mathrm{O}-\mathrm{H}$ bond-stretching vibrational modes and is related to the different stacking of atomic layers.

DOI: 10.1103/PhysRevX.5.021033

Subject Areas: Condensed Matter Physics, Materials Science, Physical Chemistry
\end{abstract}

\section{INTRODUCTION}

Sixfold symmetric snow crystals are formed from hexagonal ice (Ih), which covers about $10 \%$ of Earth's surface and plays a prominent role in determining its climate [1-3]. A cubic form of ice also occurs in nature, but is very rare $[4,5]$. The structures of pure hexagonal and cubic ice differ only in the stacking of layers of tetrahedrally coordinated water molecules (see Fig. 1). Yet, hexagonal ice is thermodynamically more stable than cubic ice, with experiments indicating the difference in stability to lie in the $m e V / \mathrm{H}_{2} \mathrm{O}$ range [6-15]. There is a growing realization that real "cubic ice" typically contains many stacking faults and is not pure cubic ice (Ic) as originally suggested by König [16]. Stacking faulted ice is a highly complex material, whose nature and properties depend heavily on the free-energy difference between pure Ih and Ic.

The very similar free energies of Ih and Ic have so far prevented state-of-the-art first-principles quantum mechanical calculations from explaining the stability of Ih. Both density functional theory (DFT) and diffusion quantum Monte Carlo studies have found that Ih and Ic are almost degenerate in energy when nuclear motion is neglected [18]. Our calculations show that the harmonic zero-point vibrational energies of Ih and Ic are large, at roughly $700 \mathrm{meV} / \mathrm{H}_{2} \mathrm{O}$, but they are almost identical. Consequently, when averaged over different proton orderings, the two phases are almost degenerate when harmonic vibrations are included (see Fig. 2). However, the small mass of hydrogen gives rise to large-amplitude vibrations and large anharmonic effects.

Published by the American Physical Society under the terms of the Creative Commons Attribution 3.0 License. Further distribution of this work must maintain attribution to the author(s) and the published article's title, journal citation, and DOI.
A substantial body of theoretical work exists on water and ice, based on force-field path-integral molecular dynamics, first-principles classical molecular dynamics, and first-principles vibrational calculations in the quasiharmonic approximation. This work has led to significant successes in understanding the important role of quantum vibrations and anharmonicity for various phenomena observed experimentally. Examples include, but are not limited to, (a) the isotope effects, e.g., on the melting temperature, of Ih upon going from protiated to deuterated ice [20-22], (b) accurate $\mathrm{O}-\mathrm{H}$ bond lengths and infrared $\mathrm{O}-\mathrm{H}$ stretching frequencies in water [23-25], (c) reproduction of the anomalous thermal expansion, and the isotope effect on the volume in Ih [26], and (d) the heat capacity of water [27]. Attempts to calculate the relative stability of Ih and Ic have either relied on empirical force fields such as TIP4P $[28,29]$ or have lacked an accurate description of anharmonicity $[18,30]$. TIP4P has since been shown to produce incorrect proton-ordering energetics and an incorrect static lattice energy difference between Ih and Ic compared to highly accurate diffusion Monte Carlo methods [31]. Moreover, no successful attempts to explain the origin of the greater stability of Ih have been made. With our fully anharmonic, first-principles DFT study we show that the inclusion of accurate anharmonic quantum nuclear motion is decisive in stabilizing Ih with respect to Ic, and relate the difference in stability to the different stacking of the atomic layers.

The water molecules in both Ih and Ic are tetrahedrally coordinated, each donating and accepting two hydrogen bonds and thus satisfying the "Bernal-Fowler ice rules" [32]. The oxygen sublattices of Ih and Ic arise from an $A B A B$ and an $A B C$ stacking of puckered layers of oxygen atoms, respectively. Correspondingly, the basic building blocks of Ih are chair- and boat-form hexamers, while Ic is built exclusively from chair-form hexamers 
(a)

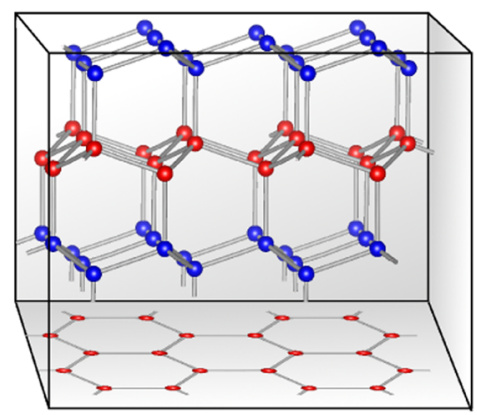

(b)

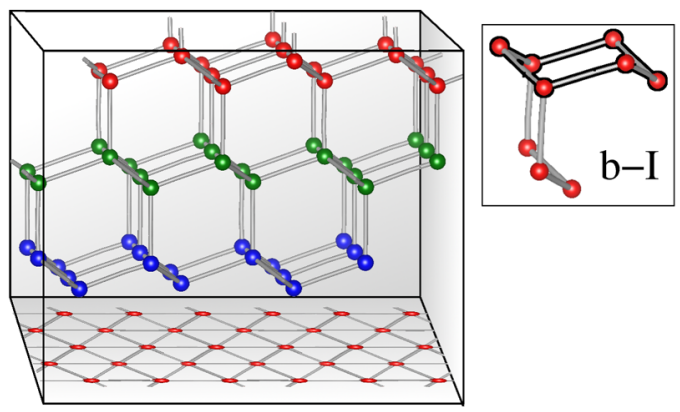

FIG. 1. The oxygen sublattices in (a) hexagonal ice Ih and (b) cubic ice Ic consist of $A B A B$ and $A B C$ stacked puckered layers (red, blue, and green colors), respectively. Projections of the periodic structures along the direction orthogonal to the puckered layers are shown on the lower faces of the diagrams. Ih is built of the chair- and boat-form hexamers shown in a-I and a-II, respectively, while Ic is built exclusively from chair-form hexamers as in b-I. The structures were visualised using VESTA [17].

(Fig. 1). Pure Ic has so far proven elusive. Experimentally synthesized "Ic" typically contains many stacking faults which strongly affect its physical and chemical properties $[6,13,33]$. Ice containing cubic sequences interlaced with hexagonal sequences is commonly known as stacking-disordered ice (Isd). Unlike Ih and Ic, which refer to a unique stacking arrangement of puckered layers, Isd refers to the infinite set of possible stacking sequences. This set smoothly connects $\mathrm{Ih}$ as one end member to Ic as the other. Isd has trigonal $P 3 m 1$ symmetry [34-36]. As of yet, it is unclear whether stacking disorder is kinetically or thermodynamically driven. A full understanding of Isd will require understanding the properties of Ih and Ic, including their relative stability. At the most basic level, the free-energy difference between Ih and Ic is required to understand why Isd is found to anneal to Ih rather than Ic.

The fraction of cubic stackings of layers, or "cubicity," typically does not exceed around 60\% [33] in experiments, and both the fraction itself as well as the nature of the stacking arrangements depend heavily on the synthesis pathway $[13,33,37]$. Crucially, ice synthesized via both homogeneous and heterogeneous freezing of (supercooled) water has a random stacking of cubic and hexagonal layers $[33,37]$, which is consistent with a layer-by-layer growth

mechanism. Heterogeneous freezing in particular is central to atmospheric and climate physics and, due to random stacking disorder, depends vitally on the free-energy difference between Ih and Ic. It is clear that Isd is an extremely important and highly complex material.

Molecular dynamics and Monte Carlo simulations using empirical ice potentials to model ice nucleation processes have successfully reproduced stacking disorder (see Refs. [29,37,38] and references therein). However, they struggle, among other issues, with the accuracy of the empirical potentials in describing the melting temperature and the relative stability of Ih and Ic [39].

In the following we limit ourselves to the study of pure Ih and Ic.

\section{COMPUTATIONAL MODEL}

Atomistic simulations of proton-disordered systems such as Ih and Ic require sets of explicit atomic positions, and a calculation must be performed for each proton ordering studied. The number of proton-ordered, energetically quasidegenerate structures allowed by the ice rules increases exponentially with the size of the simulation cell. This leads to Pauling's residual configurational entropy [40] $S_{\text {config }}$, which has been confirmed experimentally [41,42]. For large systems with negligible surface effects, the associated configurational free energies of Ih and Ic $\Delta G_{\text {config }}=-T S_{\text {config }}$ are almost identical $[43,44]$. We therefore neglect $\Delta G_{\text {config }}$ in the following.

To gain an understanding of the effects of proton ordering on the vibrational properties of ice, we consider 16 distinct proton-ordered eight-molecule Ih configurations as constructed by Hirsch and Ojamäe [45], and 11 distinct proton-ordered eight-molecule Ic configurations [18]. We also consider the "conventional" hexagonal, 12-molecule $\mathrm{P6}_{3} \mathrm{~cm}$ Ih and quasicubic, eight-molecule $P 4_{3}$ Ic structures (numbers 13 and 1 in Fig. 2, respectively). Details of the proton-ordered structures and their numbering are provided in Supplemental Table I [19].

We perform electronic structure calculations using plane-wave pseudopotential DFT as implemented in the CASTEP code [46] (version 7.02). We employ the PerdewBurke-Ernzerhof (PBE) [47] generalized gradient approximation to the exchange-correlation functional, and on-the-fly generated ultrasoft pseudopotentials [48] with core radii of 0.7 and $0.8 \AA$ for the hydrogen and oxygen atoms, respectively. Supplemental Sec. V describes results obtained with other density functionals [19]. We use a plane-wave energy cutoff of $1600 \mathrm{eV}$ and Monkhorst-Pack reciprocal space grids of spacing less than $2 \pi \times 0.04 \AA^{-1}$ for all total energy calculations and geometry optimizations. The resulting energy differences between frozen-phonon configurations are converged to below $10^{-4} \mathrm{eV} / \mathrm{H}_{2} \mathrm{O}$, the atomic positions to within $10^{-5} \AA$, and the residual forces to within $10^{-4} \mathrm{eV} / \AA$. 


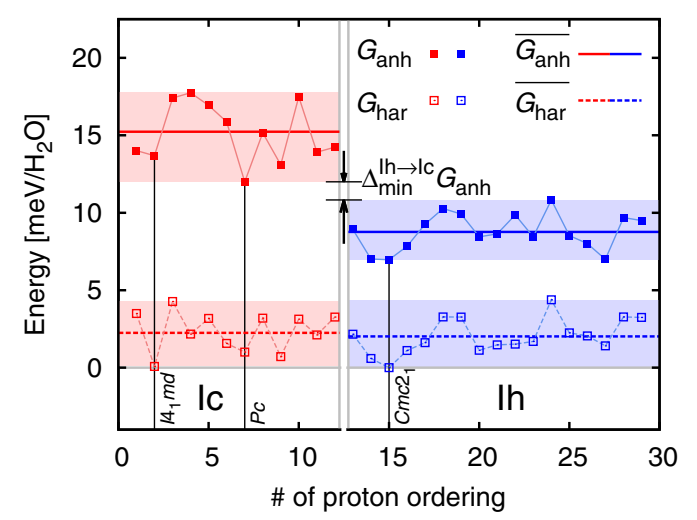

FIG. 2. Harmonic ( $G_{\text {har }}$, empty squares, lower part) and anharmonic ( $G_{\text {anh }}$, filled squares, upper part) energies including zero-point nuclear motion for different Ih (blue) and Ic (red) proton orderings (Supplemental Table I [19]). The averages over proton orderings $\overline{G_{\mathrm{har}}}$ and $\overline{G_{\mathrm{anh}}}$ are shown as thick horizontal dotted and solid lines, respectively. All energies are measured with respect to $G_{\text {har }}^{\mathrm{XIh}}$ (Ih $C m c 2_{1}$, structure 15).

We obtain harmonic vibrational free energies from the harmonic frequencies calculated using the $\mathbf{k}$-space Fourierinterpolated dynamical matrix. The latter is obtained by Fourier transforming the real-space matrix of force constants constructed using a finite displacement method. Anharmonic vibrational free energies are calculated using the method described in Ref. [49], which has so far been successfully applied to high-pressure systems [50,51]. As in Ref. [49], we describe the $3 \mathcal{N}$-dimensional BornOppenheimer (BO) energy surface (where $\mathcal{N}$ is the number of atoms in the simulation cell) by mapping $1 \mathrm{D}$ subspaces along the harmonic normal mode axes up to large amplitudes of 4 times the harmonic root-mean-square (rms) displacements, where anharmonicity is important. We then reconstruct the $3 \mathcal{N}$-dimensional $\mathrm{BO}$ surface from the $1 \mathrm{D}$ subspaces. The resultant representation of the BO energy surface is an approximation to the true $3 \mathcal{N}$-dimensional BO energy surface. This approximation only weakly affects the free-energy difference between Ih and Ic (see Supplemental Sec. VI [19]). The 1D energy surfaces are fitted using cubic splines. The anharmonic vibrational Schrödinger equation is solved within a vibrational self-consistent field framework. The vibrational wave function is expanded in a basis of simple harmonic oscillator eigenstates, and the inclusion of 25 states for each vibrational degree of freedom is found sufficient to obtain converged results.

\section{RESULTS}

Our calculations show that the static lattice energies of Ih and Ic $E_{\text {static }}$ vary by up to $5 \mathrm{meV} / \mathrm{H}_{2} \mathrm{O}$ with proton ordering. This agrees with Refs. [18,45,52] and, more importantly, with Ref. [53], which evaluates DFT static lattice energies for 16 eight-molecule orthorhombic, 14 twelve-molecule hexagonal, and 63 forty-eight-molecule orthorhombic Ih proton orderings. This strongly suggests that our sets of proton orderings provide a good representation of the distribution of energies in disordered ice.

We also find that the harmonic vibrational contributions to the free energies of different proton orderings $\Delta G_{\text {har }}$ vary by up to $2 \mathrm{meV} / \mathrm{H}_{2} \mathrm{O}$. We have employed the vibrational self-consistent field method described above to calculate the anharmonic contribution to the vibrational free energy $\Delta G_{\text {anh }}$, finding a variation between proton orderings of up to $5 \mathrm{meV} / \mathrm{H}_{2} \mathrm{O}$.

The free energies of Ih and Ic at the harmonic vibrational level $G_{\text {har }}=E_{\text {static }}+\Delta G_{\text {har }}$, averaged over the proton orderings, are virtually indistinguishable:

$$
\Delta_{\mathrm{av}}^{\mathrm{Ic} \rightarrow \mathrm{Ih}} G_{\mathrm{har}} \equiv \overline{G_{\mathrm{har}}^{\mathrm{Ic}}}-\overline{G_{\mathrm{har}}^{\mathrm{Ih}}}=0.2 \pm 2.4 \mathrm{meV} / \mathrm{H}_{2} \mathrm{O},
$$

where the quoted errors are the rms variations across different proton orderings. The anharmonic energies of the Ih configurations $\Delta G_{\text {anh }}$, on the other hand, though also positive, are systematically lower than those of the Ic configurations, so that the total free energy $G_{\text {anh }}=$ $E_{\text {static }}+\Delta G_{\text {har }}+\Delta G_{\text {anh }}$, averaged over the different proton orderings, is significantly lower for Ih than for Ic:

$$
\Delta_{\mathrm{av}}^{\mathrm{Ic} \rightarrow \mathrm{Ih}} G_{\mathrm{anh}} \equiv \overline{G_{\mathrm{anh}}^{\mathrm{Ic}}}-\overline{G_{\mathrm{anh}}^{\mathrm{Ih}}}=6.5 \pm 3.1 \mathrm{meV} / \mathrm{H}_{2} \mathrm{O} .
$$

The values obtained for $\Delta_{\mathrm{av}}^{\mathrm{Ic} \rightarrow \mathrm{Ih}} G_{\mathrm{har}}$ and $\Delta_{\mathrm{av}}^{\mathrm{Ic} \rightarrow \mathrm{lh}} G_{\mathrm{anh}}$ depend significantly on the method of averaging. For example, using a Boltzmann distribution for the free energy of the proton orderings leads to values of $\Delta_{\text {Boltzmann }}^{\mathrm{Ic} \rightarrow \mathrm{Ih}} G_{\mathrm{anh}} \approx 5.5$ and $6.1 \mathrm{meV} / \mathrm{H}_{2} \mathrm{O}$ at 10 and $100 \mathrm{~K}$, respectively.

It is noteworthy that, given cell volumes that are reasonably close to experiment, the differences in $E_{\text {static }}$, $\Delta G_{\text {har }}$, and $\Delta G_{\text {anh }}$ between Ih and Ic depend only weakly on the details of the DFT calculations and in particular on the choice of exchange-correlation functional (see Fig. 3 and Supplemental Sec. V [19]).

The most stable proton-ordered configurations of Ih and Ic, referred to as XIh and XIc, display free-energy differences very similar to $\Delta_{\mathrm{av}}^{\mathrm{Ic} \rightarrow \mathrm{Ih}} G_{\mathrm{har}}$ and $\Delta_{\mathrm{av}}^{\mathrm{Ic} \rightarrow \mathrm{Ih}} G_{\mathrm{anh}}$. However, the inclusion of anharmonic vibrational energies changes the relative stability of the different proton orderings. XIh is experimentally and theoretically known to have space group $C m c 2_{1}[45,61]$ (structure 15 in Fig. 2), a result confirmed by our calculations. A structure of space group $I 4_{1} m d$ has been proposed [18] (structure 2) for XIc on theoretical grounds. Our calculations support this proposal at the harmonic vibrational level, but the inclusion of anharmonic contributions suggests that Ic $P c, P c a 2_{1}$, and $P_{n a 2}$ (structures 7, 9, and 12) may also be strong candidates for XIc. Reference [62] reports experimental evidence for Ic $I 4_{1} m d$ and $P n a 2_{1}$ in partially protonordered Ic via Fourier-transform infrared spectroscopy. This lends significant support to our result that anharmonicity provides the decisive contribution to the energy 


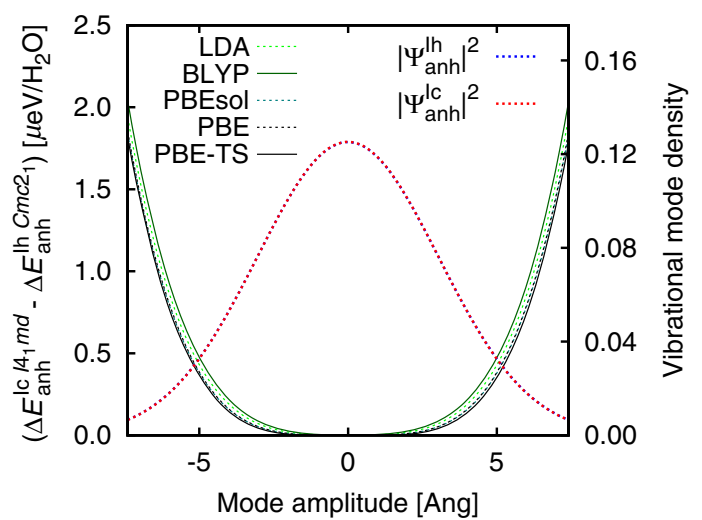

FIG. 3. Difference in the anharmonicity of the BO energy surface $\Delta E_{\text {anh }}$ of Ih $C m c 2_{1}$ and Ic $I 4_{1} m d$ for the LDA [54-56], BLYP [57,58], PBEsol [59], PBE [47] and PBE-TS [60] functionals (thin solid and dotted lines). The dominant contribution to the anharmonicity is quartic. The difference between the results from different functionals is so small it can barely be distinguished. The different widths of the vibrational densities $\left|\Psi_{\text {anh }}\right|^{2}$ of Ih and Ic (blue and red thick dotted lines, respectively) is indistinguishable on the scale of the figure.

differences between proton orderings, since $P n a 2_{1}$ has a high free energy at the static lattice and harmonic vibrational levels and only becomes a low-free-energy structure when anharmonicity is taken into account.

At typical experimental temperatures of below $100 \mathrm{~K}$, the proton ordering is largely frozen in. Consequently, one cannot expect to measure a change in free energy corresponding to a transition from Ic to Ih in thermal equilibrium, but rather (assuming the Ic sample is annealed at low temperatures and consists mostly of XIc) a change in free energy corresponding to transitions from XIc to a proton ordering of Ih, which is likely to be smaller than $\Delta_{\mathrm{av}}^{\mathrm{Ic} \rightarrow \mathrm{Ih}} G_{\mathrm{anh}}$. As indicated in Fig. 2, we evaluate a lower bound on the free-energy difference as

$$
\begin{aligned}
\Delta_{\min }^{\mathrm{Ic} \rightarrow \mathrm{Ih}} G_{\mathrm{anh}} & \equiv \min \left\{G_{\mathrm{anh}}^{\mathrm{Ic}}\right\}-\max \left\{G_{\mathrm{anh}}^{\mathrm{Ih}}\right\} \\
& =1.4 \pm 0.3 \mathrm{meV} / \mathrm{H}_{2} \mathrm{O} .
\end{aligned}
$$

This lower bound is consistent with, but on the high side of, experimentally measured free-energy differences of $0.3-1.6 \mathrm{meV} / \mathrm{H}_{2} \mathrm{O}$ [7-15]. Notably, the experimental value is rather uncertain, mainly because the free-energy difference is very small, and because the Ic samples are typically not fully characterized in terms of stacking faults or proton ordering.

As shown in Fig. 4, this energy difference remains roughly constant from zero temperature up to $273 \mathrm{~K}$ and thus stabilizes Ih over a wide range of temperatures.

In $\mathrm{D}_{2} \mathrm{O}$, the heavier mass of deuterium first leads to reduced vibrational frequencies of the harmonic vibrational modes and thus reduced vibrational energies. For XIh $\mathrm{D}_{2} \mathrm{O}$, $\Delta G_{\text {har }}=507.67 \mathrm{meV} / \mathrm{D}_{2} \mathrm{O}$ compared to $692.34 \mathrm{meV} / \mathrm{H}_{2} \mathrm{O}$

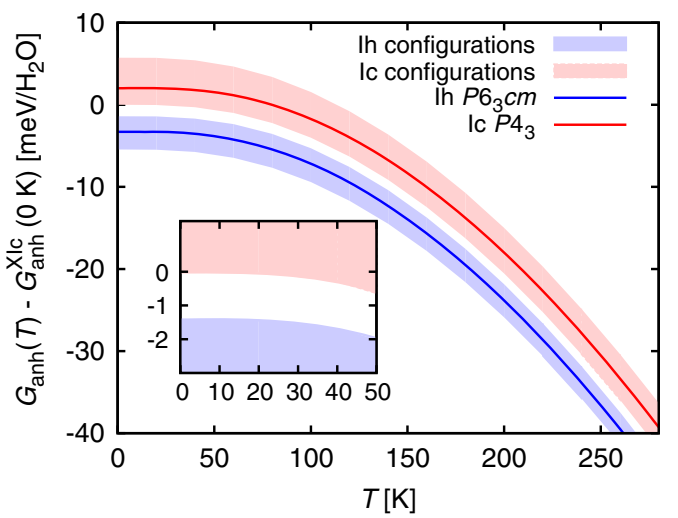

FIG. 4. $G_{\text {anh }}$ for $\mathrm{H}_{2} \mathrm{O}$ as a function of temperature. The solid blue and red lines indicate results for Ih $P 6_{3} \mathrm{~cm}$ and Ic $P 4_{3}$ simulation cells containing 96 and 64 molecules, respectively. The light blue and light red areas indicate the spread in free energies arising from the different Ih and Ic proton orderings.

for XIh $\mathrm{H}_{2} \mathrm{O}$. Second, $\mathrm{D}_{2} \mathrm{O}$ has smaller vibrational amplitudes and consequently smaller anharmonic vibrational energies than $\mathrm{H}_{2} \mathrm{O}$, resulting in a smaller difference between the free energies of Ih and Ic:

$\Delta_{\mathrm{av}}^{\mathrm{Ic} \rightarrow \mathrm{Ih}} G_{\mathrm{anh}}^{\mathrm{D}_{2} \mathrm{O}} \equiv \overline{G_{\mathrm{anh}}^{\mathrm{Ic}-\mathrm{D}_{2} \mathrm{O}}}-\overline{G_{\mathrm{anh}}^{\mathrm{Ih}-\mathrm{D}_{2} \mathrm{O}}}=3.7 \pm 2.9 \mathrm{meV} / \mathrm{D}_{2} \mathrm{O}$,

as shown in Fig. 5. This suggests that it could be easier to synthesize deuterated than protiated cubic ice. Moreover, the smaller anharmonic energy calculated for $\mathrm{D}_{2} \mathrm{O}$ compared with $\mathrm{H}_{2} \mathrm{O}$ implies that the most likely candidate for the ground state of cubic $\mathrm{D}_{2} \mathrm{O}$ has $I 4_{1} m d$ symmetry (structure 2) and is thus different from the ground state of $\mathrm{H}_{2} \mathrm{O}$. The predicted ground state of hexagonal $\mathrm{D}_{2} \mathrm{O}$ is $C m c 2_{1}$, as for $\mathrm{H}_{2} \mathrm{O}$.

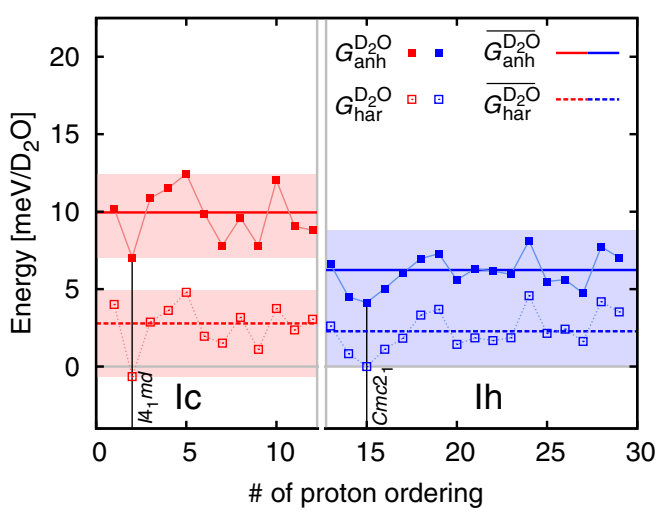

FIG. 5. Zero temperature $G_{\text {har }}^{\mathrm{D}_{2} \mathrm{O}}$ (empty squares, lower part) and $G_{\text {anh }}^{\mathrm{D}_{2} \mathrm{O}}$ (filled squares, upper part) for different deuterated Ih (blue) and Ic (red) proton orderings. The averages over proton orderings $\overline{G_{\text {har }}^{\mathrm{D}_{2} \mathrm{O}}}$ and $\overline{G_{\text {anh }}^{\mathrm{D}_{2} \mathrm{O}}}$ are shown as thick dotted and solid lines, respectively. All energies are measured with respect to $G_{\mathrm{har}}^{\mathrm{D}_{2} \mathrm{O}}$ of Ih $\mathrm{Cmc2} 2_{1}$. 


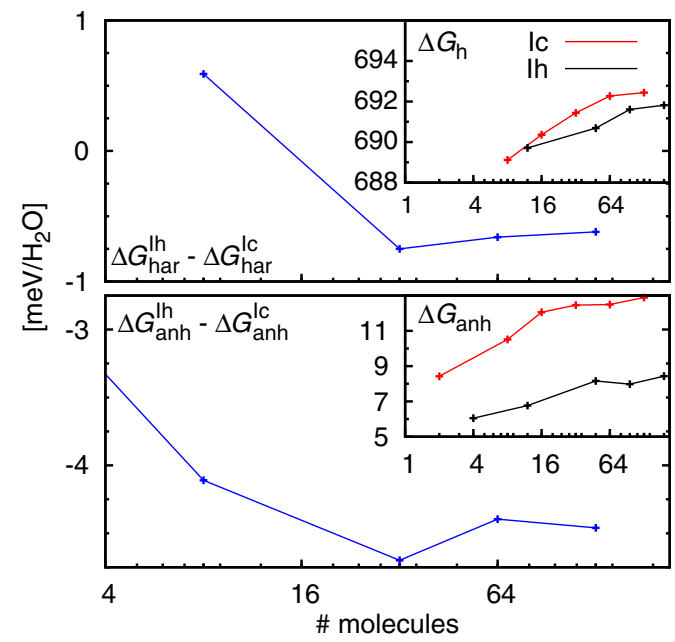

FIG. 6. The harmonic free energy $\Delta G_{\text {har }}$ and anharmonic free energy $\Delta G_{\text {anh }}$ are shown as functions of the simulation cell size in the upper and lower panels, respectively.

We have investigated the convergence of $\Delta G_{\text {har }}$ and $\Delta G_{\text {anh }}$ with respect to the size of the simulation cell using cells containing up to 192 molecules for Ih $P 6_{3} \mathrm{~cm}$ and 128 molecules for Ic $P 4_{3}$ as shown in Fig. 6.

(a)

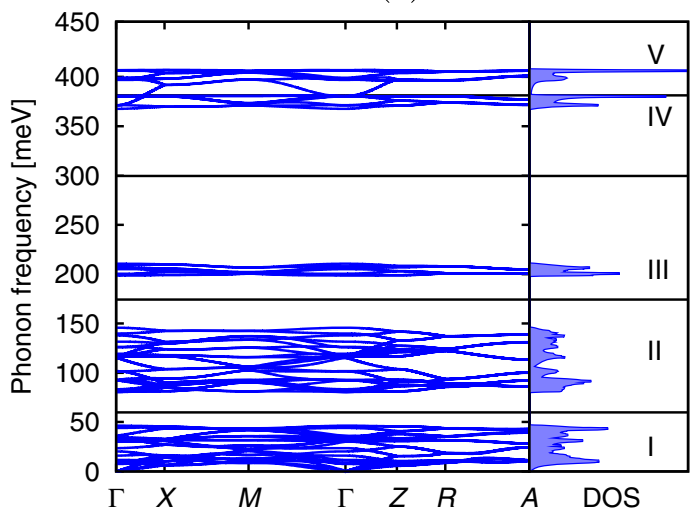

(b)

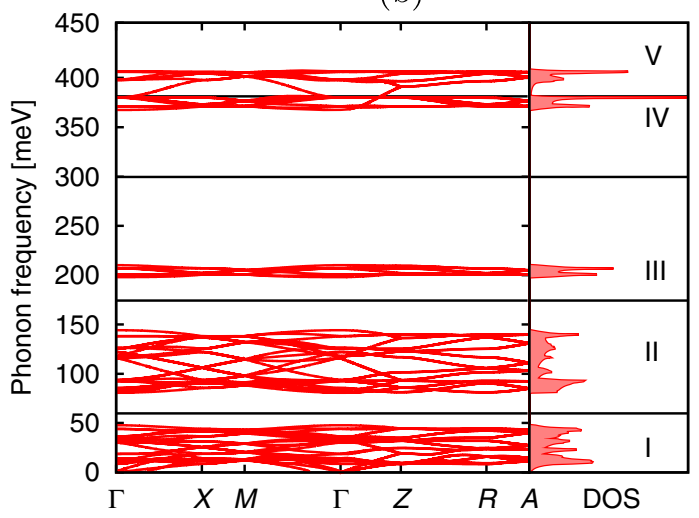

FIG. 7. Vibrational band structure and DOS of (a) Ih and (b) Ic split into crystal modes (I), libration modes (II), molecular $\mathrm{O}-\mathrm{H}$ bending modes (III), and molecular $\mathrm{O}-\mathrm{H}$ antisymmetric and symmetric stretching modes (IV and V, respectively).
The vibrational energies of the different proton orderings are calculated using 64-molecule cells for the harmonic vibrational energy and eight-molecule cells for the anharmonic energies. The latter is justified by the short-range nature of anharmonicity, evidence for which is described in Sec. IV. Calculations using cells with up to 192 molecules for the $P 6_{3} \mathrm{~cm}$ Ih structure and 128 molecules for the $P 4_{3}$ Ic structure indicate that the difference between $\Delta G_{\text {har }}$ for Ih and Ic is converged to within $0.1 \mathrm{meV} / \mathrm{H}_{2} \mathrm{O}$ using 64-molecule simulations cells. The anharmonic corrections converge analogously, and the difference between $\Delta G_{\text {anh }}$ for the two structures is converged to within $0.2 \mathrm{meV} / \mathrm{H}_{2} \mathrm{O}$ using 64-molecule simulations cells. More details may be found in Supplemental Sec. II [19].

\section{DISCUSSION}

The origin of the difference in the anharmonicities of Ih and Ic can be traced back to the libration $(80-140 \mathrm{meV})$ and, predominantly, the molecular symmetric and antisymmetric $\mathrm{O}-\mathrm{H}$ bond-stretching modes $(365-380 \mathrm{meV}$ and

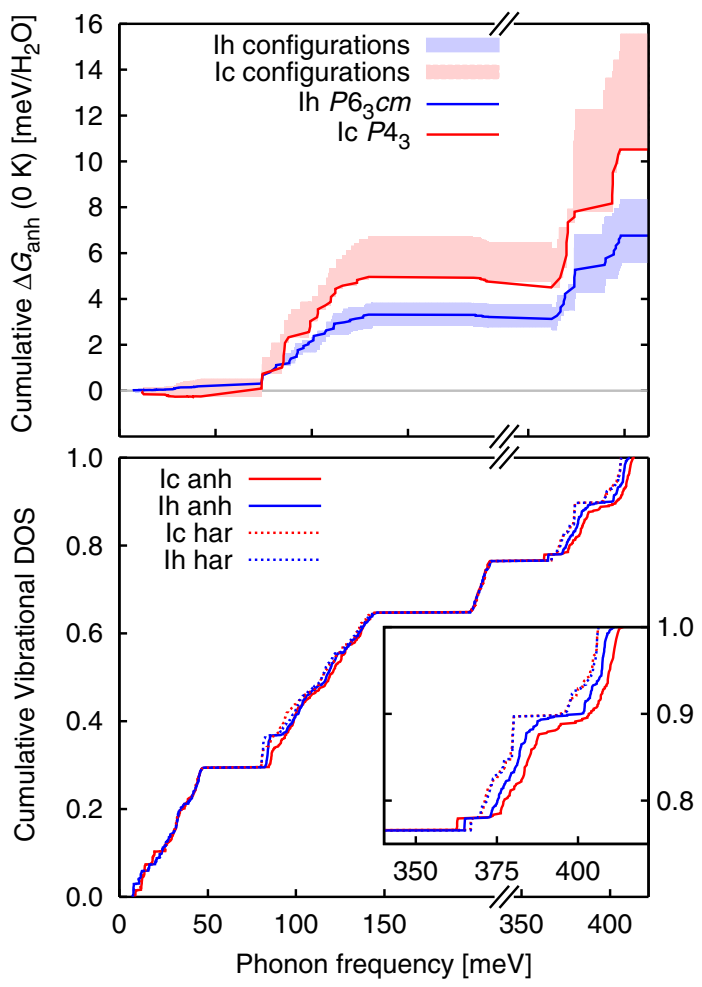

FIG. 8. Cumulative anharmonic energies and vibrational DOS of $\mathrm{H}_{2} \mathrm{O}$ averaged over proton orderings as a function of frequency. The upper panel shows the anharmonic energies for Ih $C m c 2_{1}$ and Ic $P 4_{3}$ as solid blue and red lines and the spread in free energies in Ih and Ic due to different proton orderings as light red and light blue areas. The lower panel shows that the harmonic DOS of Ih and Ic (dotted lines) are practically identical. The larger increase in vibrational energy due to anharmonicity in Ic than in Ih is reflected in the shifts of their anharmonic DOS (continuous lines) with respect to the harmonic DOS. 
395-410 meV, respectively) indicated in Fig. 7. The vibrational density of states (DOS) and the distribution of anharmonic corrections over the vibrational frequencies show (Fig. 8) that the dominant anharmonic contributions arise from the $\mathrm{O}-\mathrm{H}$ bond-stretching modes, which correspond to large-amplitude displacements of the hydrogen atoms relative to their neighboring (essentially stationary) oxygen atoms. The comparatively small role of the oxygen atoms is confirmed by studying Ih and Ic analogues with fixed oxygen positions, which recover the value of $\Delta G_{\text {anh }}$ observed for real Ih and Ic to within 5\% (see Supplemental Table III [19]). The O-H bond-stretching modes contribute $>2 / 3$ of the difference in anharmonicity between Ih and Ic, when averaged over proton orderings. Note that the energy difference between Ih and Ic shown in Fig. 8 increases at high frequencies.

(a)

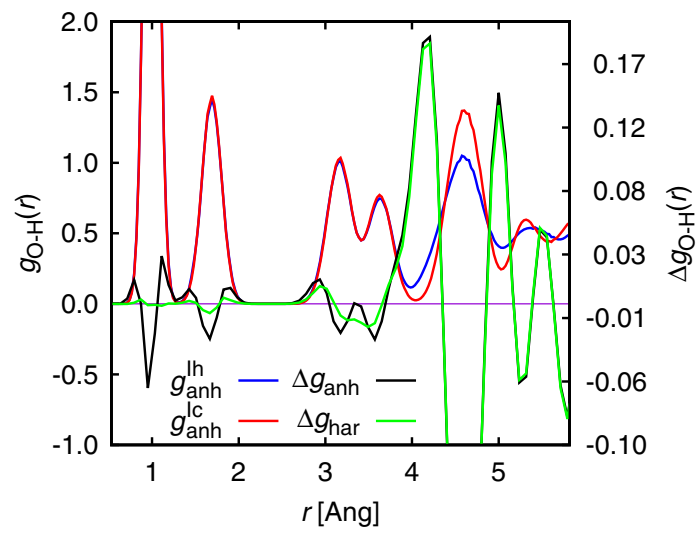

(b)

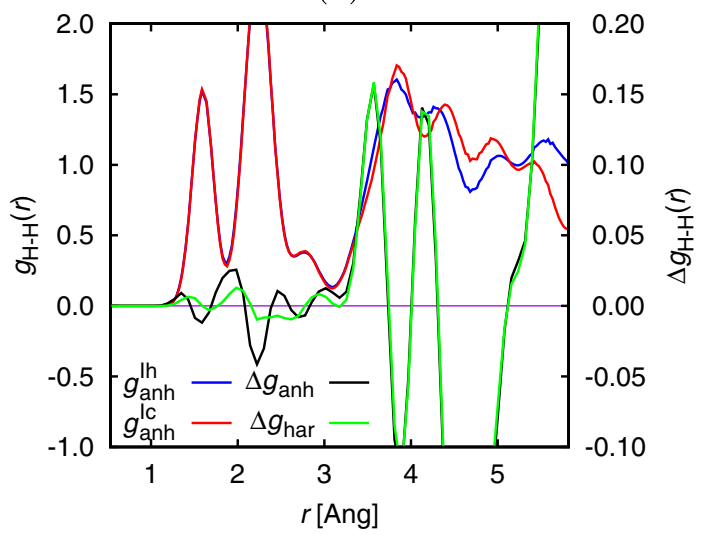

FIG. 9. The O-H RDFs, $g_{\mathrm{O}-\mathrm{H}(r)}$, (a) and H-H RDFs, $g_{\mathrm{H}-\mathrm{H}(r)}$, (b) for Ih $P 6_{3} \mathrm{~cm}$ (blue lines) and Ic $P 4_{3}$ (red lines) are sampled from the anharmonic nuclear wave functions. For radii $<3 \AA$ the difference between Ih and Ic at the harmonic level (green lines) $\Delta g_{\mathrm{har}} \equiv g_{\mathrm{har}}^{\mathrm{Ih}}-g_{\mathrm{har}}^{\mathrm{Ic}}$ is very small. The difference at the anharmonic level (black lines) $\Delta g_{\mathrm{anh}} \equiv g_{\mathrm{anh}}^{\mathrm{Ih}}-g_{\mathrm{anh}}^{\mathrm{Ic}}$ is small, but nonnegligible. The features in $\Delta g_{\text {har }}$ and $\Delta g_{\text {anh }}$ for radii beyond around $3 \AA$ originate predominantly from differences in the static structures of Ih and Ic.
Variations in vibrational frequencies with cubicity have recently been identified in infrared absorption experiments [6]. Carr et al. observed that the $\mathrm{O}-\mathrm{H}$ stretching modes were shifted to higher frequencies with increasing cubicity of the Isd samples. They also observed an increasing broadening of the absorption peak. According to Carr et al., both trends are thought to be associated with the stacking disorder, which peaks at a cubicity of 50\%. For samples with cubicities of 50\%, Carr et al. observed shifts of $28 \pm 2$ and $13 \pm 2 \mathrm{~cm}^{-1}$ for protiated and deuterated Isd, respectively. Our calculations reproduce the widening of the $\mathrm{O}-\mathrm{H}$ stretching peak in the vibrational DOS and produce shifts of $70 \pm 5$ and $30 \pm 5 \mathrm{~cm}^{-1}$ for protiated and deuterated Isd,

(a)

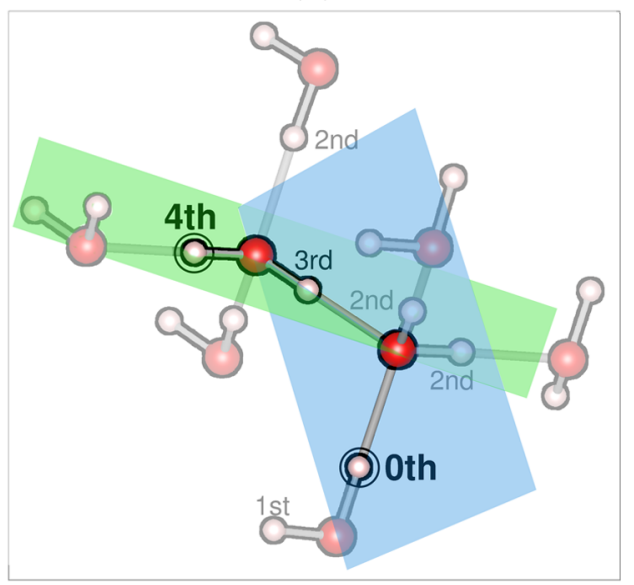

(b)

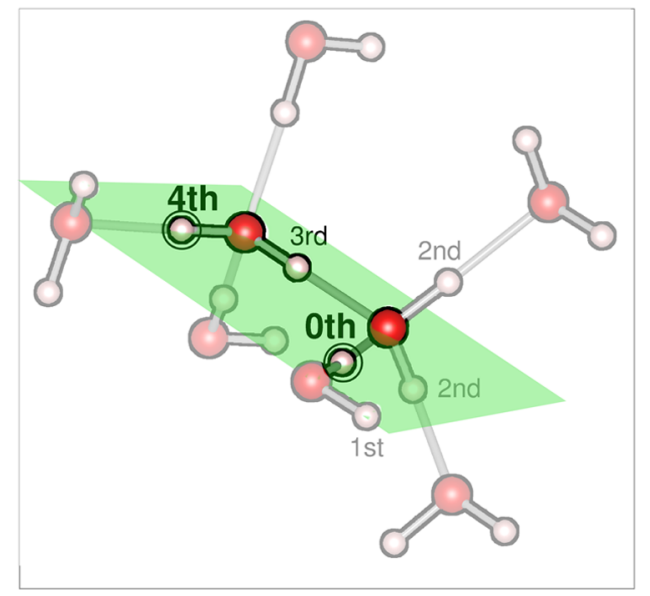

FIG. 10. The (arbitrarily picked) central proton is labeled 0th. The nearest- to fourth-nearest-neighbors are labeled 1st through 4th. From the point of view of the central proton, the fourthnearest-neighbor protons first lead to a distinction between chairand boat-form hexamers. The fourth-nearest-neighbor protons are found at distances from the central proton ranging from around 3.5 to $5.0 \AA$ A. The green and blue planes are spanned by the relevant $\mathrm{O}-\mathrm{O}$ axes connecting the fourth-nearest-neighbor protons. (a) Typical chair-form configuration found in Ih and Ic. (b) Typical boat-form configuration found only in Ih. 
respectively; i.e., at about double the cubicity of Carr etal., we calculate shifts that are about twice as large as those of Carr et al. Thus, our values are consistent with the results of Carr et al. if the shift scales roughly linearly with the degree of cubicity rather than the amount of stacking disorder, and is largest in pure Ic. In accurately reproducing the ratio between the blueshifts of the molecular stretching frequencies for the protiated and deuterated phases, our results also provide a good account of the effects of isotopic substitution.

The role of the high-energy modes can be further illuminated by considering the $\mathrm{H}-\mathrm{H}$ radial distribution functions (RDFs) [Fig. 9(b)] of Ih and Ic and rms displacements of the protons (see Supplemental Table IV [19]). The latter show that the harmonic vibrational amplitudes in Ic are about $1 \%$ smaller than in Ih. Yet, at the harmonic level the $\mathrm{H}-\mathrm{H}$ radial distribution functions of $\mathrm{Ih}$ and Ic are essentially identical for distances less than about $3 \AA$. Beyond $3 \AA$ the static structures of the two phases differ. Unlike the rms displacements of the protons, the RDFs measure a two-particle quantity that describes correlated motions of pairs of protons. At the harmonic level the protons in Ic move less with respect to their equilibrium positions than in Ih, but they move by just as much with respect to each other. We note that the $\mathrm{O}-\mathrm{H}$ and $\mathrm{H}-\mathrm{H}$ RDFs for Ih shown in Figs. 9(a) and 9(b) agree well with, e.g., the experimental RDFs in Ref. [63].

While the protons in both phases feel the same local environment, differences occur starting with the fourthnearest-neighbor protons (see Fig. 10). Also, for systems as small as 8 to 12 molecules $\Delta G_{\text {anh }}$ is already about $3 / 4$ of the converged value (Supplemental Fig. 1 [19]). This system size limits the wavelength of the vibrational modes responsible for the difference in anharmonic energies to roughly the same distance as the separation of fourthnearest-neighbor protons. Together, these observations indicate that the influence of more distant nuclei is small. Allowing for both chair- and boat-form hexamers, there are 12 distinct arrangements of fourth-nearest-neighbor pairs of protons. Out of these 12 arrangements only 8 are realized in the proton orderings we have considered. These are shown in Fig. 11. Three of these arrangements (numbers VI-VIII) are associated with boat-form hexamers of $\mathrm{H}_{2} \mathrm{O}$ molecules, which only exist in $\mathrm{Ih}$, and five (numbers $\mathrm{I}-\mathrm{V}$ ) are associated with the chair-form hexamers of $\mathrm{H}_{2} \mathrm{O}$ molecules found in Ih and Ic. The RDFs in Fig. 11 show that, on going from Ic to Ih, arrangements II and IV are depopulated. For arrangements II and IV the displacement of the first proton of the pair from its equilibrium position along its hydrogen bridge bond leads to a large displacement relative to the second proton. Conversely, arrangements VI-VIII, for which the same displacement of the first proton leads to a far smaller displacement relative to the second, are populated. This explains why on average the protons in Ic move less with respect to their equilibrium positions than in Ih, while moving by just as much with respect to each other, resulting in the same $G_{\text {har }}$ as in Ih.

Going beyond the harmonic approximation, anharmonicity reduces the rms vibrational amplitudes in Ih and Ic by around $1 \%$ and $2.5 \%$, respectively, localizing the nuclear wave function more in ice Ic than Ih. On the level of the collective vibrational modes, the localization is typically driven by a strong quartic contribution to the respective $\mathrm{BO}$
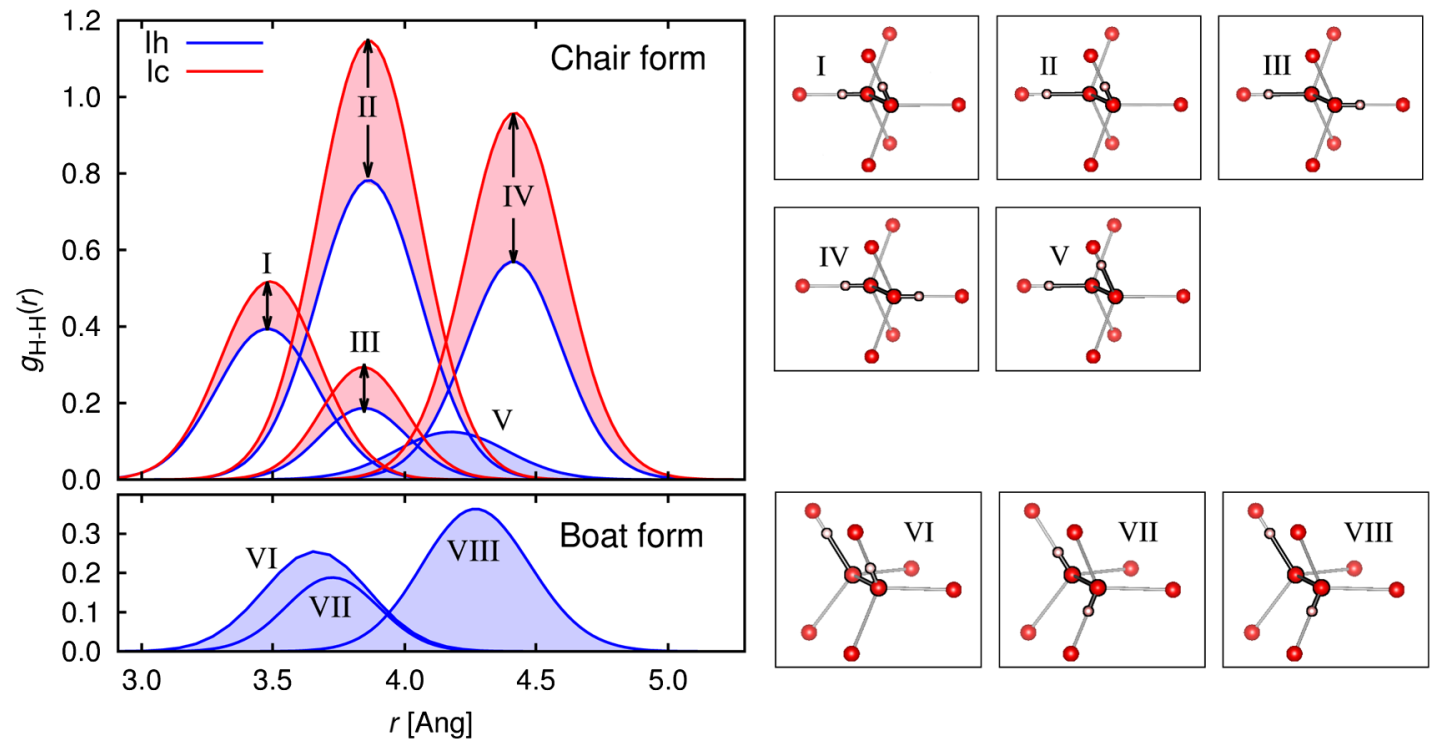

FIG. 11. Anharmonic H-H RDF decomposed into contributions from different bonding configurations of fourth-nearest-neighbor pairs of protons. The upper part shows the components corresponding to the chair-form hexamer configurations I-V found in both Ih and Ic. The lower part shows the components corresponding to the boat-form hexamer configurations VI-VIII, which are exclusive to Ih. In configurations VI-VIII the hydrogen bridge bonds associated with the pairs of protons (shown as small white spheres) are at a larger angle to each other than in I-V. 
energy surfaces (see Supplemental Fig. 6 [19]). Moreover, at the anharmonic level the RDFs show that the protons in ice Ic move less with respect to each other than in Ih, instead of just moving less with respect to their equilibrium positions, as they do at the harmonic level. This difference in the relative motion of pairs of protons is the origin of the difference in $\Delta G_{\text {anh }}$ between Ih and Ic. The larger effect of anharmonicity in Ic is again due to the stronger geometric "coupling" between pairs of protons.

\section{PERSPECTIVE}

Ih and Ic are important in various branches of science. Examples include climate modeling and the simulation of ice nucleation and formation, where cubic ice plays an important role and for which $\Delta G_{\text {anh }}$ is an essential input parameter. As an example of the relevance in biological sciences, the benign shape of cubic ice crystals is of potential interest for cryopreservation [64]. Here we have demonstrated that accounting for anharmonic nuclear vibrations is central to understanding and correctly predicting the free-energy difference between Ih and Ic. However, the importance of anharmonic vibrations in hydrogen-bonded systems reaches far beyond ice. An accurate treatment of anharmonicity is likely to be crucial in correctly describing the energy differences between very similar polymorphs of hydrogen-bonded molecular crystals which are important in, e.g., pharmaceutical materials science.

Calculating anharmonic vibrational energies in solids is a challenging computational task, which has only recently been successfully achieved using first-principles quantum mechanical methods. Anharmonic effects are particularly important for light elements, such as the hydrogen atoms in $\mathrm{H}_{2} \mathrm{O}$. Anharmonic vibrations are also expected to be important at the surfaces of ice, and when impurities or other defects are present.

\section{ACKNOWLEDGMENTS}

We acknowledge financial support from the Engineering and Physical Sciences Research Council of the U.K. [EP/ J017639/1]. B. M. also acknowledges Robinson College, Cambridge, and the Cambridge Philosophical Society for a Henslow Research Fellowship. The calculations were performed on the Cambridge High Performance Computing Service facility and the HECToR and Archer facilities of the U.K.'s national high-performance computing service (for which access was obtained via the UKCP consortium [EP/K013564/1]).

[1] T. Bartels-Rausch, V. Bergeron, J. H. E. Cartwright, R. Escribano, J. L. Finney, H. Grothe, P. J. Gutiérrez, J. Haapala, W. F. Kuhs, J. B. C. Pettersson, S. D. Price, C. I. Sainz-Díaz, D. J. Stokes, G. Strazzulla, E. S. Thomson,
H. Trinks, and N. Uras-Aytemiz, Ice structures, patterns, and processes: A view across the ice-fields, Rev. Mod. Phys. 84, 885 (2012).

[2] M. B. Baker and T. Peter, Small-scale cloud processes and climate, Nature (London) 451, 299 (2008).

[3] K. C. Young, Microphysical Processes in Clouds (Oxford University Press, New York, 1993).

[4] B. J. Murray, D. A. Knopf, and A. K. Bertram, The formation of cubic ice under conditions relevant to the earth's atmosphere, Nature (London) 434, 202 (2005).

[5] J. E. Shilling, M. A. Tolbert, O. B. Toon, E. J. Jensen, B. J. Murray, and A. K. Bertram, Measurements of the vapor pressure of cubic ice and their implications for atmospheric ice clouds, Geophys. Res. Lett. 33, L17801 (2006).

[6] T. H. G. Carr, J. J. Shephard, and C. G. Salzmann, Spectroscopic signature of stacking disorder in ice I, J. Phys. Chem. Lett. 5, 2469 (2014).

[7] K. Thürmer and S. Nie, Formation of hexagonal and cubic ice during low-temperature growth, Proc. Natl. Acad. Sci. U.S.A. 110, 11757 (2013).

[8] O. Yamamuro, M. Oguni, T. Matsuo, and H. Suga, Heat capacity and glass structure of pure and doped cubic ices, J. Phys. Chem. Solids 48, 935 (1987).

[9] Y. P. Handa, D. D. Klug, and E. Whalley, Difference in energy between cubic and hexagonal ice, J. Chem. Phys. 84, 7009 (1986).

[10] E. Mayer and A. Hallbrucker, Cubic ice from liquid water, Nature (London) 325, 601 (1987).

[11] J. A. McMillan and S. C. Los, Vitreous ice: irreversible transformations during warm-up, Nature (London) 206, 806 (1965).

[12] M. Sugisaki, H. Suga, and S. Seki, Calorimetric study of the glassy state IV: Heat capacities of glassy water and cubic ice, Bull. Chem. Soc. Jpn. 41, 2591 (1968).

[13] W. F. Kuhs, C. Sippel, A. Falenty, and T. C. Hansen, Extent and Relevance of Stacking Disorder in "Ice Ic", Proc. Natl. Acad. Sci. U.S.A. 109, 21259 (2012).

[14] B. J. Murray, S. L. Broadley, T. W. Wilson, S. J. Bull, R. H. Wills, H. K. Christenson, and E. J. Murray, Kinetics of the homogeneous freezing of water, Phys. Chem. Chem. Phys. 12, 10380 (2010).

[15] D. C. Steytler, J. C. Dore, and C. J. Wright, Neutron diffraction study of cubic ice nucleation in a porous silica network, J. Phys. Chem. 87, 2458 (1983).

[16] H. König, A cubic modification of ice, Z. Kristallogr. 105, 279 (1944).

[17] F. Izumi and K. Momma, Three-dimensional visualization in powder diffraction, Solid State Phenom. 130, 15 (2007).

[18] Z. Raza, D. Alfè, C. G. Salzmann, J. Klimeš, A. Michaelides, and B. Slater, Proton ordering in cubic ice and hexagonal ice; a potential new ice phase-XIc, Phys. Chem. Chem. Phys. 13, 19788 (2011).

[19] See Supplemental Material at http://link.aps.org/ supplemental/10.1103/PhysRevX.5.021033 for more information on the choice of proton ordered structures, the convergence of the free energies with respect to the simulation cell size, the rms vibrational displacements of the nuclei, the choice of the exchange-correlation functional, and the effects of quantum zero-point and thermal expansion. 
[20] R. Ramírez and C. P. Herrero, Quantum path integral simulation of isotope effects in the melting temperature of ice Ih, J. Chem. Phys. 133, 144511 (2010).

[21] C. P. Herrero and R. Ramírez, Isotope effects in ice Ih: A path-integral simulation, J. Chem. Phys. 134, 094510 (2011).

[22] L. Hernández de la Peña, M. S. Gulam Razul, and P. G. Kusalik, Quantum effects in ice Ih, J. Chem. Phys. 123, 144506 (2005).

[23] S. Habershon, T. E. Markland, and D. E. Manolopoulos, Competing quantum effects in the dynamics of a flexible water model, J. Chem. Phys. 131, 024501 (2009).

[24] A. Zeidler, P. S. Salmon, H. E. Fischer, J. C. Neuefeind, J. M. Simonson, and T. E. Markland, Isotope effects in water as investigated by neutron diffraction and path integral molecular dynamics, J. Phys. Condens. Matter 24, 284126 (2012).

[25] C. P. Herrero and R. Ramírez, High-density amorphous ice: A path-integral simulation, J. Chem. Phys. 137, 104505 (2012).

[26] B. Pamuk, J. M. Soler, R. Ramírez, C. P. Herrero, P. W. Stephens, P. B. Allen, and M. V. Fernandez-Serra, Anomalous Nuclear Quantum Effects in Ice, Phys. Rev. Lett. 108, 193003 (2012).

[27] C. Vega, M. M. Conde, C. McBride, J. L. F. Abascal, E. G. Noya, R. Ramírez, and L. M. Sesé, Heat capacity of water: A signature of nuclear quantum effects, J. Chem. Phys. 132, 046101 (2010).

[28] H. Tanaka and I. Okabe, Thermodynamic stability of hexagonal and cubic ices, Chem. Phys. Lett. 259, 593 (1996).

[29] D. Quigley, Communication: Thermodynamics of stacking disorder in ice nuclei, J. Chem. Phys. 141, 121101 (2014).

[30] R. Ramírez, N. Neuerburg, and C. P. Herrero, The phase diagram of ice: A quasi-harmonic study based on a flexible water model, J. Chem. Phys. 139, 084503 (2013).

[31] S. J. Singer and C. Knight, in Advances in chemical physics, edited by S. A. Rice and A. R. Dinner (Wiley, Hoboken, NJ, 2011), Vol. 147.

[32] J. D. Bernal and R. H. Fowler, A Theory of water and ionic solution, with particular reference to hydrogen and hydroxyl ions, J. Chem. Phys. 1, 515 (1933).

[33] T. L. Malkin, B. J. Murray, C. G. Salzmann, V. Molinero, S. J. Pickering, and T. F. Whale, Stacking disorder in ice I, Phys. Chem. Chem. Phys. 17, 60 (2015).

[34] T. C. Hansen, M. M. Koza, and W. F. Kuhs, Formation and annealing of cubic ice: I. Modelling of stacking faults, J. Phys. Condens. Matter 20, 285104 (2008).

[35] T. C. Hansen, M. M. Koza, P. Lindner, and W. F. Kuhs, Formation and annealing of cubic ice: II. kinetic study, J. Phys. Condens. Matter 20, 285105 (2008).

[36] J. Hallett, W. P. Arnott, M. P. Bailey, and J. T. Hallett, in Cirrus, edited by K. D. Lynch, K. Sassen, D. O’C. Starr, and G. Stephens (Oxford University Press, Oxford, 2002).

[37] E. B. Moore, E. de la Llave, K. Welke, D. A. Scherlis, and V. Molinero, Freezing, melting and structures of ice in a hydrophilic nanopore, Phys. Chem. Chem. Phys. 12, 4124 (2010).
[38] D. L. Silva, Jr. and M. de Koning, Structure and energetics of extended defects in ice Ih, Phys. Rev. B 85, 024119 (2012).

[39] L. Hernández de la Peña and P. G. Kusalik, Quantum effects in liquid water and ice: Model dependence, J. Chem. Phys. 125, 054512 (2006).

[40] L. Pauling, The structure and entropy of ice and of other crystals with some randomness of atomic arrangement, J. Am. Chem. Soc. 57, 2680 (1935).

[41] Y. Tajima, T. Matsuo, and H. Suga, Phase transition in KOH-Doped hexagonal ice, Nature (London) 299, 810 (1982).

[42] S. M. Jackson and R. W. Whitworth, Thermally-stimulated depolarization studies of the ice XI-Ice Ih phase transition, J. Phys. Chem. B 101, 6177 (1997).

[43] J. F. Nagle, Lattice statistics of hydrogen bonded crystals. The residual entropy of ice, J. Math. Phys. (N.Y.) 7, 1484 (1966).

[44] C. P. Herrero and R. Ramírez, Configurational entropy of hydrogen-disordered ice polymorphs, J. Chem. Phys. 140, 234502 (2014).

[45] K. Hirsch and L. Ojamäe, Quantum-chemical and force-field investigations of ice Ih: Computation of proton-ordered structures and prediction of their lattice energies, J. Phys. Chem. B 108, 15856 (2004).

[46] S. J. Clark, M. D. Segall, C. J. Pickard, P. J. Hasnip, M. I. J. Probert, K. Refson, and M. C. Payne, First principles methods using CASTEP, Z. Kristallogr. 220, 567 (2005).

[47] J. P. Perdew, K. Burke, and M. Ernzerhof, Generalized Gradient Approximation Made Simple, Phys. Rev. Lett. 77, 3865 (1996).

[48] D. Vanderbilt, Soft self-consistent pseudopotentials in a generalized eigenvalue formalism, Phys. Rev. B 41, 7892 (1990).

[49] B. Monserrat, N. D. Drummond, and R. J. Needs, Anharmonic vibrational properties in periodic systems: Energy, electron-phonon coupling, and stress, Phys. Rev. B 87, 144302 (2013).

[50] B. Monserrat, N. D. Drummond, C. J. Pickard, and R. J. Needs, Electron-Phonon Coupling and the Metallization of Solid Helium at Terapascal Pressures, Phys. Rev. Lett. 112, 055504 (2014).

[51] S. Azadi, B. Monserrat, W. M. C. Foulkes, and R. J. Needs, Dissociation of High-Pressure Solid Molecular Hydrogen: A Quantum Monte Carlo and Anharmonic Vibrational Study, Phys. Rev. Lett. 112, 165501 (2014).

[52] J. Lekner, Energetics of hydrogen ordering in ice, Physica (Amsterdam) 252B, 149 (1998).

[53] S. J. Singer, J. L. Kuo, T. K. Hirsch, C. Knight, L. Ojamäe, and M. L. Klein, Hydrogen-Bond Topology and the Ice VII/ VIII and Ice Ih/XI Proton-Ordering Phase Transition, Phys. Rev. Lett. 94, 135701 (2005).

[54] D. M. Ceperley and B. J. Alder, Ground State of the Electron Gas by a Stochastic Method, Phys. Rev. Lett. 45, 566 (1980).

[55] J. P. Perdew and A. Zunger, Self-interaction correction to density-functional approximations for many-electron systems, Phys. Rev. B 23, 5048 (1981). 
[56] J. P. Perdew and Y. Wang, Accurate and simple analytic representation of the electron-gas correlation energy, Phys. Rev. B 45, 13244 (1992).

[57] A. D. Becke, Density-functional exchange-energy approximation with correct asymptotic behavior, Phys. Rev. A 38, 3098 (1988).

[58] C. Lee, W. Yang, and R. G. Parr, Development of the ColleSalvetti correlation-energy formula into a functional of the electron density, Phys. Rev. B 37, 785 (1988).

[59] J. P. Perdew, A. Ruzsinszky, G. I. Csonka, O. A. Vydrov, G. E. Scuseria, L. A. Constantin, X. Zhou, and K. Burke, Restoring the Density-Gradient Expansion for Exchange in Solids and Surfaces, Phys. Rev. Lett. 100, 136406 (2008); J. P. Perdew, A. Ruzsinszky, G. I. Csonka, O. A. Vydrov, G. E. Scuseria, L. A. Constantin, X. Zhou, and K. Burke, Erratum: Restoring the Density-Gradient Expansion for Exchange in Solids and Surfaces, Phys. Rev. Lett. 102, 039902(E) (2009).
[60] A. Tkatchenko and M. Scheffler, Accurate Molecular Van Der Waals Interactions from Ground-State Electron Density and Free-Atom Reference Data, Phys. Rev. Lett. 102, 073005 (2009).

[61] S. M. Jackson, V. M. Nield, R. W. Whitworth, M. Oguro, and C. C. Wilson, Single-crystal neutron diffraction studies of the structure of ice XI, J. Phys. Chem. B 101, 6142 (1997).

[62] P. Geiger, C. Dellago, M. Macher, C. Franchini, G. Kresse, J. Bernard, J. N. Stern, and T. Loerting, Proton ordering of cubic ice Ic: Spectroscopy and computer simulations, J. Phys. Chem. C 118, 10989 (2014).

[63] A. Soper, The radial distribution functions of water and ice from 220 to $673 \mathrm{~K}$ and at pressures up to $400 \mathrm{MPa}$, Chem. Phys. 258, 121 (2000).

[64] P. Mehl and P. Boutron, Cryoprotection of red blood cells by 1,3-Butanediol and 2,3-Butanediol, Cryobiology 25, 44 (1988). 